\title{
GCU
}

Glasgow Caledonian

University

University for the Common Good

\section{Monitoring insulator contamination level under dry condition with a microwave reflectometer}

Jiang, Yan; McMeekin, Scott; Reid, Alistair; Nekahi, Azam; Judd, Martin; Wilson, Alan

Published in:

2015 IEEE 11th International Conference on the Properties and Applications of Dielectric Materials (ICPADM)

DOI:

10.1109/ICPADM.2015.7295433

Publication date:

2015

Document Version

Author accepted manuscript

Link to publication in ResearchOnline

Citation for published version (Harvard):

Jiang, Y, McMeekin, S, Reid, A, Nekahi, A, Judd, M \& Wilson, A 2015, Monitoring insulator contamination level under dry condition with a microwave reflectometer. in 2015 IEEE 11th International Conference on the Properties and Applications of Dielectric Materials (ICPADM). IEEE, pp. 959 - 962.

https://doi.org/10.1109/ICPADM.2015.7295433

\section{General rights}

Copyright and moral rights for the publications made accessible in the public portal are retained by the authors and/or other copyright owners and it is a condition of accessing publications that users recognise and abide by the legal requirements associated with these rights.

Take down policy

If you believe that this document breaches copyright please view our takedown policy at https://edshare.gcu.ac.uk/id/eprint/5179 for details

of how to contact us. 


\title{
Monitoring Insulator Contamination Level under Dry Condition with a Microwave Reflectometer
}

\author{
Yan Jiang, Scott G. McMeekin, Alistair J. Reid and \\ Azam Nekahi \\ School of Engineering and Built Environment \\ Glasgow Caledonian University \\ Glasgow, United Kingdom
}

\author{
Martin D. Judd \\ High Frequency Diagnostics and Engineering Ltd \\ Glasgow, United Kingdom
}

\author{
Alan Wilson \\ Doble PowerTest Ltd \\ Surrey, United Kingdom
}

\begin{abstract}
Build-up of surface contamination on high voltage insulators can lead to an increase in leakage current and partial discharge, which may eventually develop into flashover. Conventional contamination level monitoring systems based on leakage current, partial discharge, infrared and ultraviolet camera are only effective when the contamination layer has been wetted by rain, fog or condensation; under these conditions flashover might occur before there is time to implement remedial measures such as cleaning. This paper describes studies exploring the feasibility of applying microwave reflectometry techniques to monitor insulator contamination levels. This novel method measures the power generated by a $10.45 \mathrm{GHz}$ source and reflected at the insulator contamination layer. A theoretical model establishes the relationship between equivalent salt deposit density (ESDD) levels, dielectric properties and geometry of contamination layers. Experimental results demonstrate that the output from the reflectometer is able to clearly distinguish between samples with different contamination levels under dry conditions. This contamination monitoring method could potentially provide advance warning of the future failure of wet insulators in climates where insulators can experience dry conditions for extended periods.
\end{abstract}

Keywords - Insulators; Microwave Reflectometry; Insulator contamination; Pollution measurement

\section{INTRODUCTION}

High voltage insulators are employed extensively in overhead transmission lines and substations and form an essential component in power systems and networks. Outdoor insulators can experience a variety of harsh operating conditions and environments. Insulators located near coastal regions encounter sodium chloride $(\mathrm{NaCl})$ in the form of sea water while those located in inland areas may be contaminated by road salt and industrial pollutants such as paper pulp, fly-ash, cement dust, etc [1]. The build-up of surface contamination on insulators can lead to an increase in leakage current and partial discharge, which may eventually result in flashover. Most commercial contamination monitoring systems are based on leakage current measurements using either a current transformer or a shunt resistor with an electrode ring to intercept the leakage current.
Such systems suffer from two drawbacks: (1) their physical installation onto insulator circuit would reduce insulation security, and (2) they are only effective when the contamination layer has been wetted by rain, fog or condensation; under this condition flashover is likely to occur within a short time period [2].

In order to address these problems, a novel monitoring method based on microwave reflectometry is presented in this paper. Microwave reflectometry is a non-invasive technique for analysing the properties of a medium has been widely used in radar, cable diagnostic, oceanography, non-destructive testing and medical imaging [3]. In this method, a microwave signal generated by a transmitter is reflected at the interface of interest. The reflected signal is detected by a receiver. Properties of the reflected signal such asmagnitude, time delay and power level contain the information concerning the object under test. In this paper, the power level of the reflected signal is investigated. The contamination layer on a polluted insulator reflects a different electromagnetic energy level compared to a clean insulator under the same microwave illumination. Thus, the contamination level on an insulator surface can be determined by measuring the received power level of the reflected signal.

In the present paper, both a theoretical model and experiments have been implemented to study the feasibility of this method. The theoretical model evaluates the relationship between ESDD levels, dielectric properties and geometry of contamination layers. Experiments have been carried out under laboratory conditions using artificial polluted samples. A constant $10.45 \mathrm{GHz}$ signal generated by a transmitter is reflected at the contamination layer and the insulator surface and then detected by a highly sensitive receiver.

\section{THEORETICAL MODEL}

For a contamination layer on an insulator, the reflected power is affected by material properties and geometry. The material properties contain the complex permittivity and permeability of the contamination which are related to ESDD. The geometry contains the thickness and roughness of the 
contamination layer, shape of the insulator and the locations and shapes of both transmitter and receiver antennas.

Fig. 1 shows the structure of the proposed system model relating outputs of the receiver to the ESDD on insulator surface. Because the composition of the contamination on insulator surface is similar to the soil, obtaining the complex permittivity and permeability of the contamination on insulator might therefore be treatable in a similar manner to determine soil properties. Within this framework, the dielectric mixing model evaluates the complex permittivity and permeability of insulator contamination layer as a function of moisture, salinity, environment temperature and humidity by assuming it as salt and water affected soil. The FDTD model uses Finitedifference time-domain (FDTD) simulation to evaluate the complex geometries of antennas, insulator and contamination layer. Finally, the receiver model calculates the output voltage of the receiver which is inversely proportional to the reflected power.

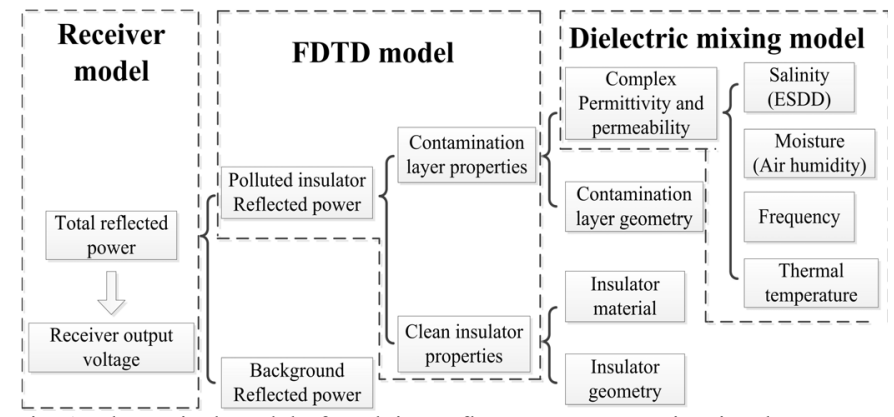

Fig. 1. Theoretical model of applying reflectometry to monitor insulator contamination.

\section{A. Dielectric mixing model}

The dielectric mixing model developed by Dobson [4] is a classical model for remotely sensing soil moisture. It gives the complex permittivity of soil as a function of its water content. In consideration of the effect of the salt, this dielectric mixing model needs to be modified as it only applies to very low salt content. After replacing the dielectric constant model for water with the dielectric constant model of saline water, developed by Stogryn [5], the dielectric mixing model will carry the information of both moisture and salinity. On the insulator surface, moisture is a function of air humidity, which can be easily measured. The composition of the contamination layer is based on the IEC60507.

In these models, complex permittivity $\varepsilon$ is a complex number $\varepsilon=\varepsilon^{\prime}-j \varepsilon^{\prime \prime}$ with real part $\varepsilon^{\prime}$ and imaginary part $\varepsilon^{\prime \prime}$. The real part $\varepsilon^{\prime}$ is related to the stored energy within the medium and the imaginary part $\varepsilon^{\prime \prime}$ is related to the dissipation (or loss) of energy within the medium. The model will be developed from pure water to saline water and finally to the salt-affected soil, which is intended to approximate a contamination layer on an insulator surface.

In terms of Stogryn's formulation, the real and imaginary parts of the complex permittivity of saline water are respectively given by $[6,7]$ :

$$
\left\{\begin{array}{c}
\varepsilon_{S w}^{\prime}=\varepsilon_{S w \infty}+\frac{\varepsilon_{S w 0}-\varepsilon_{S w \infty}}{1+\left(2 \pi f \tau_{s w}\right)^{2}} \\
\varepsilon_{S w}^{\prime \prime}=\frac{2 \pi f \tau_{S w}\left(\varepsilon_{s w 0}-\varepsilon_{S w \infty}\right)}{1+\left(2 \pi f \tau s_{w}\right)^{2}}+\frac{\sigma_{N a C l}}{2 \pi \varepsilon_{0} f}
\end{array}\right.
$$

Where, $\varepsilon_{w 0}$ is the static dielectric constant; $\varepsilon_{w \infty}$ is the highfrequency dielectric constant; $\varepsilon_{s w \infty}=\varepsilon_{w \infty}=4.9$ and $\varepsilon_{0}=$ $8.854 \times 10^{-12}(\mathrm{~F} / \mathrm{m})$ is the permittivity of free space. $\tau_{s w}$ is the relaxation time of the saline water; $\sigma_{\mathrm{NaCl}}$ is the conductivity of $\mathrm{NaCl}$ solution and $f$ is the frequency.

Dobson's model defined in (2) below represents the dielectric constant of soil as a function of soil moisture $m_{v}$, dielectric constant of free water inside soil $\varepsilon_{f w}$, permittivity of dry soil $\varepsilon_{s}$, bulk density of dry soil $\rho_{s}$ and bulk density of wet soil $\rho_{b}$.

$$
\left\{\begin{array}{c}
\varepsilon_{m}^{\prime}=\left(1+\left(\rho_{b} / \rho_{s}\right) \varepsilon_{s}^{\alpha}+m_{v}^{\beta^{\prime}} \varepsilon_{f w}^{\prime \alpha}-m_{v}\right)^{\frac{1}{\alpha}} \\
\varepsilon_{m}^{\prime \prime}=\left(m_{v}^{\beta^{\prime \prime}} \cdot \varepsilon_{f w}^{\prime \prime \alpha}\right)^{\frac{1}{\alpha}}
\end{array}\right.
$$

where, $\alpha$ and $\beta$ are related to the soil texture. By combining (2) with Stogryn's model (1), free water is considered as saline water where: $\varepsilon_{f w}^{\prime}=\varepsilon_{s w}^{\prime}$ and $\varepsilon_{f w}^{\prime \prime}=\varepsilon_{s w}^{\prime \prime}$. In IEC standard 60507 , the contamination layer consists entirely of Kaolin, a form of clay with a typical bulk density of $\rho_{b}=1 \mathrm{~g} / \mathrm{cm}^{3}$. The complex permeability of the contamination can be expressed by:

$$
\mu_{m}=1 /\left(\mu_{0} \varepsilon_{0} \varepsilon_{m} v_{p}^{2}\right)
$$

where $v_{p}$ is the phase velocity of light; $\varepsilon_{m}$ is the complex permittivity of the contamination layer and $\mu_{0}$ is the permeability of free space.

\section{B. FDTD model}

A 3-dimensional finite difference time domain (FDTD) model was built to simulate the electromagnetic behaviors of the whole transmitter-receiver system. The simulation had the same setup as the subsequent experiment. As shown in Fig. 2, it was decided to use flat glass samples with dimensions of 500 $\mathrm{mm} \times 200 \mathrm{~mm}$ and $8 \mathrm{~mm}$ thickness to remove the effect of the complex surface geometry of the HV insulators for the purpose of testing the concept. The flat sample was covered with a 0.5 $\mathrm{mm}$ thick homogeneous flat contamination layer. Two X-band horn antennas were pointing to the flat sample with an angle $\theta$. A $10.45 \mathrm{GHz}$ sinusoidal signal was injected to the left-side horn antenna while the total power received by the right-side horn antenna was recorded by a plane sensor. The sample is $600 \mathrm{~mm}$ away from each antenna and placed on the focus of two antennas as shown in Fig. 2..

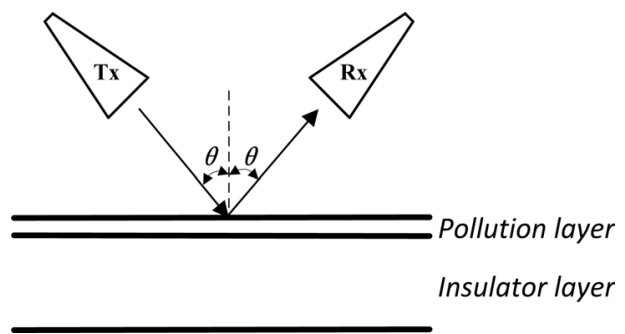

Fig. 2. The geometry of the FDTD model. (Tx: transmitter, Rx: Receiver) 
The material properties used for the contamination layer were the complex permittivity and permeability calculated by dielectric mixing model at $10.45 \mathrm{GHz}$ with 6 different ESDD level under dry conditions. The glass plane was assigned constant permittivity and permeability. The antennas were treated as perfect electrical conductors. All the boundaries were defined using absorbing boundary conditions to eliminate unwanted microwave reflections into the region of interest. The angle of the antennas $\theta$ was varied from $10^{\circ}$ to $75^{\circ}$ with a $5^{\circ}$ step to find the angle offering highest sensitivity. Each simulation ran $40 \mathrm{~ns}$ with 4 ps time step.

Fig. 3 shows the simulated power received when $\theta=20^{\circ}$ and ESDD level is $0.12 \mathrm{mg} / \mathrm{cm}^{2}$. It shows that the wave travels from the transmitter to sample and finally reaches the receiver after 5 ns. The received signal takes another 15 ns to stabilize. The output of the receiver dependents on the average power after the system becomes stable. Fig. 4 shows the relationship between sensitivity of the system and the angle of the antennas.

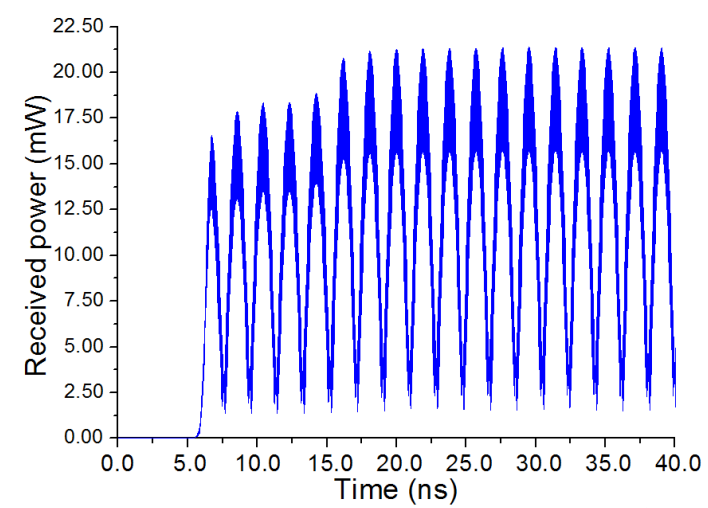

Fig. 3. Power on receiver antenna $\left(\theta=20^{\circ}, \mathrm{ESDD}=0.12 \mathrm{mg} / \mathrm{cm}^{3}\right)$

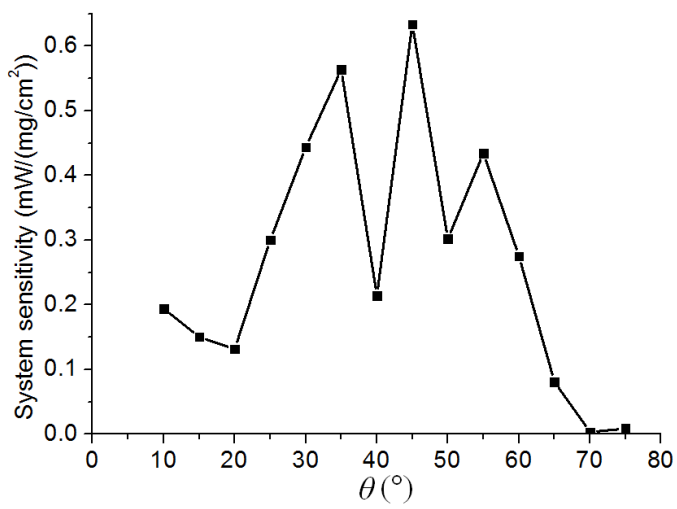

Fig. 4. Relationship between sensitivity of the system and the angle of the antennas

According to Fig. 4, system sensitivity of antenna received power to ESDD level on sample surface strongly relates to the angle of the antennas, reaching a maximum when $\theta=45^{\circ}$ but with notches at neighboring angles. The sensitivity changes rapidly with small angle changes around $45^{\circ}$ so for the initial experimental setup, $30^{\circ}$ was chosen due to its high sensitivity to ESDD and lower sensitivity to the position of the antenna.

Fig. 5 shows the relationship between the average received power and the ESDD calculated by the FDTD simulation for $\theta$ $=30^{\circ}$. The results can clearly distinguish the different ESDD levels. After converting the microwave signal power into a voltage using a receiver circuit, the reflectometry may therefore be able to detect different ESDD levels under dry conditions.

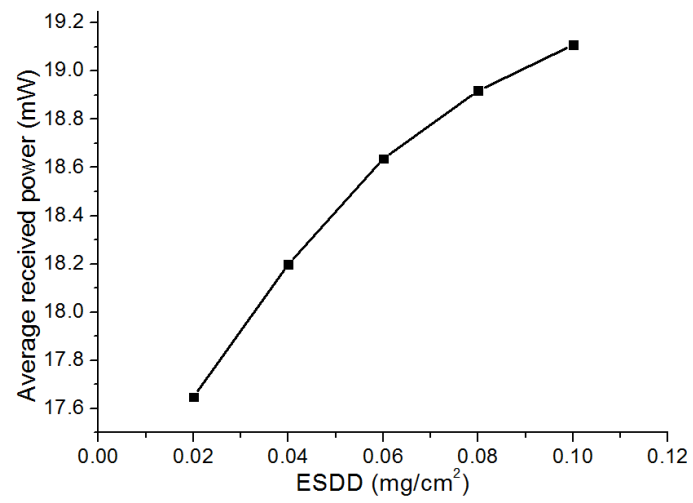

Fig. 5. Relationship between the average received power and the ESDD calculated by the FDTD simulation for $\theta=30^{\circ}$.

\section{EXPERIMENT}

\section{A. Transmitter and reciever design}

As shown in Fig. 6, the transmitter used for the experiment is an X-band horn antenna with $20 \mathrm{~dB}$ gain directly connected to a $10.45 \mathrm{GHz}$ Gunn oscillator with $+13 \mathrm{dBm}$ output power.

The receiver is a power detector with a superheterodyne architecture. After the receiver horn antenna, a low noise amplifier (LNA) amplifies the signal and a bandpass filter selects the $10.45 \mathrm{GHz}$ signal prior to the downconverter and the low-frequency circuit. The superheterodyne circuit contains a mixer and a $10.65 \mathrm{GHz}$ local oscillator. It downconverts the input signal to a $200 \mathrm{MHz}$ signal. A LNA and a bandpass filter at this frequency further improve the signal-to-noise ratio before a square-law power detector provides a dc output voltage that is inversely proportional to the input power.

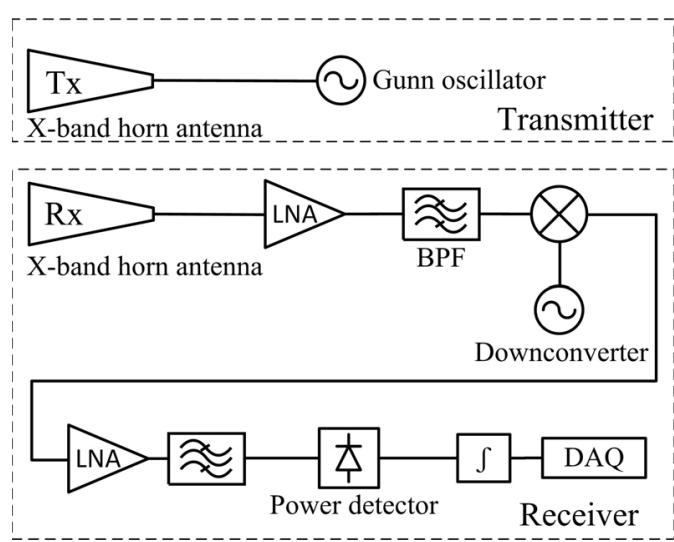

Fig. 6. Transmitter and receiver circuits

\section{B. Setup}

To remove the effect of complex surface geometry of the $\mathrm{HV}$ insulators for the purpose of testing the concept, it was decided to use flat glass planes with the same geometry of FDTD model for the initial evaluation tests. The solid layer method recommended by IEC standard 60507 was employed to form an artificial pollution layer on the sample surfaces. This method involves uniformly spraying a pollution suspension on 
the sample surfaces to form a solid layer. The composition of the suspension used in tests comprised $6.5 \mathrm{~g}$ Kaolin, $150 \mathrm{~g}$ water and a suitable amount of $\mathrm{NaCl}$ to control the ESDD level. A $150 \mathrm{ml}$ suspension was sprayed evenly on one sample surface and the sample was then left to dry for 48 hours in a low humidity room. Four glass samples with 6 different contamination levels were tested. After the tests had been completed, the solid layers were washed off with $500 \mathrm{ml}$ distilled water and the ESDD of each sample was obtained by measuring the conductivity of the washing water. Table 1 lists the properties of the contamination layers on these sample pairs.

TABLE I. THE PROPERTIES OF THE CONTAMINATION LAYERS ON 6 SAMPLE PAIRS

\begin{tabular}{c|c|c}
\hline $\begin{array}{c}\text { Sample } \\
\text { pair }\end{array}$ & $\mathrm{NaCl}(\mathrm{g})$ & $\mathrm{ESDD}\left(\mathrm{mg} / \mathrm{cm}^{2}\right)$ \\
\hline 1 & 3 & 0.0202 \\
\hline 2 & 6 & 0.0421 \\
\hline 3 & 9 & 0.0611 \\
\hline 4 & 12 & 0.0833 \\
\hline 5 & 15 & 0.1001 \\
\hline 6 & 18 & 0.1142 \\
\hline
\end{tabular}

Both transmitter and the receiver were allowed to stabilize for 1 hour before each test to achieve thermal stability within the system. Each sample was measured 10 times with 2 seconds integration time to study the repeatability of the experiment. Within the integration time, the system output dc voltage was integrated and gave a single value to achieve better sensitivity. After each sample measurement, a clean reference sample was re-tested to evaluate the system drift. If the output voltage from the clean reference sample was found to have changed by more than $10 \mathrm{mV}$ compare to the previous test, the sample measurement would be discarded.

\section{Results}

Fig. 7 shows the relationship between the average received power and the ESDD from experiment where $\theta=30^{\circ}$. The results showed that the reflectometer output voltage decreases with the increasing of the ESDD levels on a glass sample's surface in agreement with the FDTD simulation results of Fig. 5 , since the output voltage isinversely proportional to the received power. The error bars of the results show the measurement variation over the 10 repeated tests per sample.

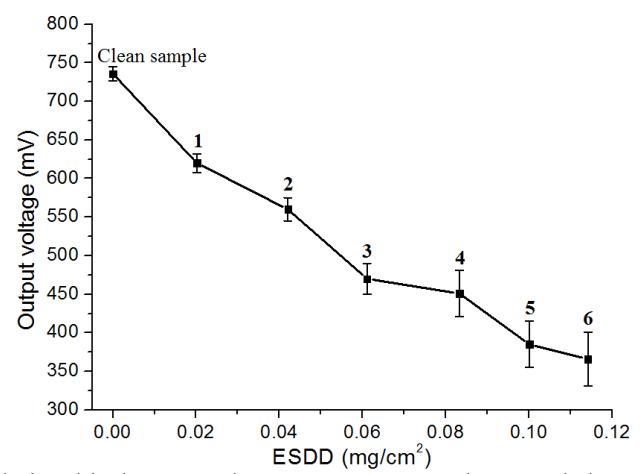

Fig. 7. Relationship between the system output voltage and the ESDD from experiment results for $\theta=30^{\circ}$.
In Fig. 7, the system presented good performance at lower ESDD levels for samples 1 and 2 on which it gave very clear differences on ESDD levels and small output drifts. However, bigger variations and overlaps of the error bars between the neighbouring samples appeared on higher ESDD level samples (e.g., 3 to 6). This is regarded as being due to crystallization of the $\mathrm{NaCl}$ in higher salinity solutions during drying generating a rough surface. Although the surface roughness brought noise for reconstructing the ESDD levels, the system could still distinguish different ESDD levels based on averaging the measurements.

\section{CONCLUSION}

This paper has presented the theoretical models and an initial experiment to study the feasibility of applying microwave reflectometry to monitor contamination levels on insulator surface. Both theoretical analysis and experimental results show this method can clearly distinguish between samples with different contamination levels. This work provides a foundation for future investigations into the development of an on-line monitoring system for insulator pollution that is effective under dry conditions.

The effect of surface roughness and the position of the antennas remains to be studied further. Because the system also receives the power reflected from other objects in the surrounding environment, a de-noising method will be necessary for on-site testing.

\section{ACKNOWLEDGMENT}

The authors would like to thank Doble Powertest and the Energy Technology Partnership (ETP) for funding this project.

\section{REFERENCES}

[1] M. A. R. M. Fernando and S. M. Gubanski, "Ageing of silicone rubber insulators in coastal and inland tropical environment", IEEE Trans. Dielectr. Electr. Insul, vol. 17, pp. 326-333, 2010.

[2] S. M. Gubanski, A. Dernfalk, J. Andersson, and H. Hillborg, "Diagnostic Methods for Outdoor Polymeric Insulators," IEEE Trans. Dielectr. Electr. Insul, vol. 14, pp. 1065-1080, 2007.

[3] S. Kharkovsky and R. Zoughi, "Microwave and millimeter wave nondestructive testing and evaluation - Overview and recent advances", IEEE Trans. Instrum. Meas. vol. 10, pp. 26-38, 2007.

[4] M. C. Dobson, F. T. Ulaby, M. T. Hallikainen, and M. A. El-Rayes, "Microwave Dielectric Behavior of Wet Soil-Part II: Dielectric Mixing Models", IEEE Trans. Geosci. Remote Sens., vol. GE-23, pp. 35-46, 1985.

[5] A. Stogryn, "Equations for Calculating the Dielectric Constant of Saline Water (Correspondence)", IEEE Trans. Microw. Theory Techn, vol. 19, pp. 733-736, 1971.

[6] J. A. Lane and J. A. Saxton, "Dielectric Dispersion in Pure Polar Liquids at Very High Radio Frequencies. III. The Effect of Electrolytes in Solution", Mathematical and Physical Sciences, vol. 214, pp. 531-545, 1952.

[7] Y. Lasne, P. Paillou, A. Freeman, T. Farr, K. C. McDonald, G. Ruffie, et al., "Effect of Salinity on the Dielectric Properties of Geological Materials: Implication for Soil Moisture Detection by Means of Radar Remote Sensing," IEEE Trans. Geosci. Remote Sens., vol. 46, pp. 1674$1688,2008$. 